Pacific Journal of Mathematics

MONOIDAL CLOSED, CARTESIAN CLOSED AND
CONVENIENT CATEGORIES OF TOPOLOGICAL SPACES 


\title{
MONOIDAL CLOSED, CARTESIAN CLOSED AND CONVENIENT CATEGORIES OF TOPOLOGICAL SPACES
}

\author{
P. BOOTH AND J. TILLOTSON
}

This paper develops a unified theory of function spaces $M_{\mathscr{T}}(Y, Z)$ with set-open topologies, the sets in question being the continuous images of selected classes of topological spaces $\mathscr{A}$. We prove that at least five of these function spaces are distinct and have corresponding exponential homeomorphisms $\theta: M_{\mathscr{N}}\left(X, M_{\mathscr{M}}(Y, Z)\right) \cong M_{\mathscr{N}}(X \times \mathscr{M}, Z)$ for suitably retopologized product spaces $X \times \leadsto Y$. Singleton spaces are normally identities with respect to these products and so we have determined four distinct monoidal closed structures for the category of all spaces. Conditions for the category of spaces generated by $\mathscr{A}$, i.e., the coreflective hull of $\mathscr{A}$, to be cartesian closed and/or convenient are given. One result asserts that the category of sequential spaces is the smallest convenient category.

R. Brown proved in [4, p. 240, Corollary 1.8] that there is an exponential homeomorphism for the category HAUS of all Hausdorff spaces, relating function spaces with the compact-open topology to a retopologized product $X \times{ }_{s} Y$ without any further restrictions on $X$ and $Y$. This product has the weak topology with respect to all subspaces of the usual product $X \times Y$ of the form:

$X \times B$ where $B$ is compact in $Y$, and $\{x\} \times Y$ where $x \in X$.

It is also shown in the same paper $[4$, p. 242 , Remark 1.15] that there is a similar general exponential law for Top relating function spaces with the topology of pointwise convergence to another suitably retopologized product. It is known [24, p. 277] that an analogous result holds for function spaces with the indiscrete topology. This is not a completely trivial example as the product used does not have the discrete topology, see example (ii) in $\S 8$ below. Hence we know of two general exponential laws for Top and, by restriction, three such for Haus. Wyler [25, p. 227] raises the question of finding new closed structures for Top. Another similar theory is developed in [21, Chapter 5] for function spaces with the cs-open (convergent sequence open) topology of $[12,13]$. It is shown ([21, p. 61] and example (i) $\S 6$ below) that the corresponding $\theta$ is a continuous bijection; we have not been able to determine if it is, in general, a homeomorphism. 
The work of [4] has been generalized by at least two authors, i.e., Vazquez [22] and Wilker [24]; the former replacing compact-open topologies by set-open topologies [1] and the latter by a type of generalized set-open topology. In each case there is an investigation of conditions that have to be placed on these defining classes of sets to ensure a reasonable exponential law.

The basic purpose of this paper is similar to that of the two authors just referred to, i.e., to develop the approach of [4] into a unified theory of function spaces with set-open topologies. Our method is to replace the use of "compact subspaces" with "maps from classes of spaces $\mathscr{A}$ ", i.e., the "sets" of our set-open topologies are the continuous images of spaces in the class $\mathscr{A}$. It has come to the authors' attention, since the completion of a first version of this manuscript, that this program was in fact proposed by $R$. Brown in [6, p. 7]. We do not, however, restrict our attention to Hausdorff space as was suggested there.

A key feature of our approach is that it leads to a theory in which the conditions arising are simple topological restrictions on the spaces in the classes $\mathscr{A}$; this has the advantage of making it very easy to generate a considerable variety of examples. Our initial restriction on the types of sets that can be used in our set-open topologies does not seem to exclude any important examples.

If $A$ is a space, $f: A \rightarrow Y$ is a map and $U$ is open in $Z$ then $W(f, U)$ will denote the subset of $M(Y, Z)$ consisting of all maps $g$ such that $g f(A) \subseteq U$.

DEFINITION 0.1. Let $\mathscr{A}$ be an arbitrary class of topological spaces. We use to topologize $M(Y, Z)$, the subbasic open sets being all sets of the form $W(f, U)$, where $A \in \mathscr{A}$ and $U$ is open in $Z$. This topology will be called the Al-open topology for $M(Y, Z)$ : the corresponding function space will be denoted by $M_{\mathscr{A}}(Y, Z)$.

It is easily seen that $M_{\mathscr{}}(-,-)$ is a bifunctor from ToP ${ }^{\mathrm{op}} \times$ ToP to ToP. This function space is similar to - but presumably not identical with - the function space $C_{a}(Y, Z)$ defined in $[25, \mathrm{p}$. 232]; the topology is a set-open topology in the sense of $[1, p .13$, Definition 4.2].

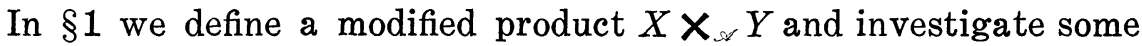
of its basic properties. We then ( $\$ 2)$ determine some conditions on $\mathscr{A}$ sufficient to ensure that $X \times{ }_{\mathscr{A}} Y$ and $M_{\mathscr{A}}(Y, Z)$ are related by an exponential law (2.4) and for a monoidal closed structure to be determined on ToP (2.6). We use 2.4 to obtain conditions under which the cartesian product $X \times Y$ and $M_{\Im}(Y, Z)$ are related by an exponential law (§3). In the particular case where $\mathscr{A}$ is the class of compact Hausdorff spaces and $X$ and $Y$ are Hausdorff this gen- 
eralizes the best known exponential law, i.e., the standard conditions relating $X \times Y$ and the compact-open topology function space $([9, \mathrm{p}$. 265, Theorem 5.3] and see example (v) below).

Given a space $X$ and a class of spaces $\mathscr{A}, a X$ will be defined to be the underlying set of $X$ retopologized with the final topology with respect to all incoming maps from spaces in $\mathscr{A}$. It is clear that $a$ is a covariant functor from Top to ToP. We call $X$ an $a$-space if $a X=X$; the category of all $a$-spaces is the coreflective hull of $\mathscr{A}$ in Top. Several authors have shown that, with suitable restrictions on $\mathscr{A}$, the category of $a$-spaces is cartesian closed. We retrieve some of these results in $\S 4$ via a different method; by simply showing

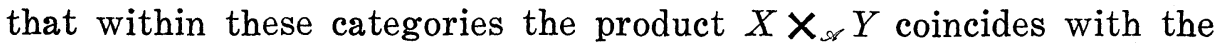
categorical products and so the cartesian closed structure is essentially a restriction of the previously established monoidal closed structure.

It is well known that the category of all $\mathfrak{k}$-spaces (= $a$-spaces with respect to the class of all compact Hausdorff spaces) is simply the category of all quotients of locally compact Hausdorff spaces, the category of all sequential spaces is the category of all quotients of first countable spaces and so on. We generalize these results in $\S 5$ to the level of arbitrary classes $\mathscr{A}$ and the corresponding $a$ spaces.

In $\S 6$ we consider the example of the $c s$-open topology and the associated cartesian closed category of sequential spaces. This leads, in $\S 7$, to a discussion of the circumstances in which cartesian closed categories of $a$-spaces can be regarded as being convenient; the point being that they must contain the underlying spaces of all $C W$ complexes, differentiable manifolds and metric spaces. Further examples are considered in $\S 8$; five of them involving function spaces with (a) the indiscrete topology, (b) the topology of pointwise convergence, (c) a topology which coincides with the compact-open topology $M_{\text {cot }}(Y, Z)$ whenever $Y$ is Hausdorff, (d) a "cube-open" topology and (e) a "compact Hausdorff countable-open" topology, are proved to generate distinct monoidal closed structures for ToP. Conditions are given in $\S 9$ for some of our function space topologies to coincide.

Notation. We use the symbols $1_{X}, 1_{X}$ etc. to denote identity maps and $i$ to denote inclusions; the symbol $\cong$ will always denote a homeomorphism. We use $U$ to denote the disjoint topological sum.

The following standard result will prove useful later.

LEMma 0.2. If . is a class of spaces containing at least one nonempty space and $X$ is an a-space then $X$ is the quotient of a topological sum of a set of spaces in. 
Proof. Let $A$ denote the set of nonclosed subsets of $X$. For each $\lambda \in \Lambda$ we can choose an $A_{\lambda} \in \mathscr{\Omega}$ and a map $p_{\lambda}: A_{\lambda} \rightarrow X$ such that $p_{\lambda}^{-1}(\lambda)$ is not closed in $A_{\lambda}$; choosing a nonempty space $A \in \mathscr{A}$ we can, for each $x \in X$, define the constant map $c_{x}: A \rightarrow X$ value $x$. Taking $\bigcup_{x \in X} A_{x}$ to be the disjoint topological sum of a collection of copies of $A$ indexed by the points of $x \in X$ then we can define

$$
p=\left(\bigcup_{\lambda \in A} p_{\lambda}\right) \cup\left(\bigcup_{x \in X} c_{x}\right):\left(\bigcup_{i \in 1} A\right) \cup\left(\bigcup_{x \in X} A_{x}\right) \longrightarrow X
$$

to be the obvious surjective map. It follows from the composition rule (or transitive law) for final topologies [5, p. 96] that $X$ has the final topology with respect to $p$ and hence is a quotient of $\left(\bigcup_{\lambda \in A} A_{\lambda}\right) \cup$ $\left(\mathbf{U}_{x \in X} A_{x}\right)$.

The authors wish to acknowledge some helpful observations by the referee, including the suggestion of 1.4(iii) and the generalization of previous versions of 4.3 and 4.4 .

\section{The $\mathscr{A}$-product.}

Definition 1.1. Given a class of spaces $\mathscr{A}$, we define $X \times \mathscr{M}$ as the set $X \times Y$ with the final topology with respect to all incoming maps of the forms:

$$
\begin{gathered}
1_{X} \times f: X \times A \longrightarrow X \times Y, f \in M(A, Y), A \in \mathscr{M} \\
i:\{x\} \times Y \longrightarrow X \times Y, x \in X .
\end{gathered}
$$

We will refer to $X \times_{\mathscr{A}} Y$ as the $\mathscr{A}$-product of $X$ and $Y$; it is clear that $-X_{s}$ - is a bifunctor from Top $\times$ ToP to Top.

Proposition 1.2. If $A$ is a class containing a nonempty space and $\left\{{ }^{*}\right\}$ denotes a singleton space then

$$
\left\{{ }^{*}\right\} \times_{\mathscr{A}} Y \cong Y \cong Y \times_{\mathscr{N}}\left\{{ }^{*}\right\} .
$$

Proof. The first homeomorphism follows from the universal property of final topologies, the second from the definition of $Y X_{\mathscr{*}}\left\{^{*}\right\}$.

Lemma 1.3. $a(Y \times Z)=a(Y \times Z)$.

Proof. The identities

$$
a(Y \times Z) \longrightarrow Y \times_{\triangleleft} Z \longrightarrow Y \times Z
$$

are clearly continuous. Applying the functor $a$, and recalling that a $a(Y \times Z)=a(Y \times Z)$, [23, Proposition 1.2(e)], the result follows. 
Proposition 1.4. Given spaces $X, Y, Z$.

(i) The identity function

$$
X \times_{s}\left(Y \times_{\mathscr{A}} Z\right) \longrightarrow\left(X \times_{s} Y\right) \times_{\star} Z
$$

is continuous.

(ii) If $\mathscr{A}$ is a class of locally compact Hausdorff spaces closed under the formation of cartesian products then the above identity is a homeomorphism.

(iii) If $\mathscr{A}$ is a class of locally compact Hausdorff spaces with the property that $A, B \in \mathscr{C}$ implies that $A \times B$ is an a-space and $X$ is a $\mathfrak{k}$-space then the above identity is a homeomorphism.

Proof. It follows from 1.3 and [23, Proposition 1.2(c)] that the natural function from $M(A, Y) \times M(A, Z)$ into $M\left(A, Y \mathrm{X}_{\mathscr{A}} Z\right)$ is a bijection, where $A \in \mathscr{A}$, hence we see, using the composition rule (or transitive law) for final topologies, that $X \times_{\mathscr{N}}\left(Y \times_{\mathscr{A}} Z\right)$ has the final topology with respect to all incoming functions

$$
\begin{aligned}
& 1_{X} \times(f, g): X \times A \longrightarrow X \times Y \times Z \\
& i \times 1_{Y} \times g:\{x\} \times Y \times A \longrightarrow X \times Y \times Z \\
& i \times i \times 1_{Z}:\{x\} \times\{y\} \times Z \longrightarrow X \times Y \times Z
\end{aligned}
$$

where $f \in M(A, Y), g \in M(A, Z), A \in \mathscr{A}, x \in X, y \in Y$.

Also we notice that $\left(X \times_{\star} Y\right) X_{\star} Z$ has the final topology with respect to all incoming functions

$$
\begin{aligned}
& 1 \times g:(X \times \propto Y) \times A \longrightarrow(X \times Y) \times Z \\
& i:\{x\} \times\{y\} \times Z \longrightarrow(X \times Y) \times Z
\end{aligned}
$$

where $g \in M(A, Z)$ and $A \in \mathscr{A}$.

(i) it is clear that $1 \times(f, g): X \times A \rightarrow X \times Y \times Z$ factors through the corresponding map $1 \times f \times g: X \times A \times A \rightarrow X \times Y \times Z$; the result follows.

Parts (ii) and (iii) require a slightly modified description of the

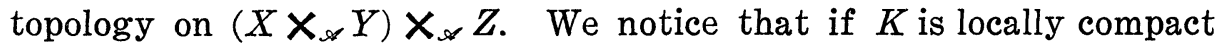
Hausdorff and $S$ has the final topology with respect to a family of incoming functions $\left\{f_{i}: C_{i} \rightarrow S\right\}_{i \in I}$ then $S \times K$ has the final topology with respect to the family $\left\{f_{i} \times 1_{K}: C_{i} \times X \rightarrow S \times K\right\}$ (this is easily verified using [9, p. 265, Theorem 5.3] and the universal property of final topologies). It follows, by the composition rule for final topologies [5, p. 96], that if $\mathscr{A}$ is a class of locally compact Haus-

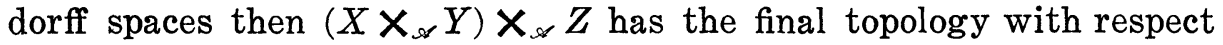
to all incoming functions 


$$
\begin{aligned}
& 1_{X} \times f \times g: X \times A^{\prime} \times A \longrightarrow X \times Y \times Z \\
& i \times 1_{Y} \times g:\{x\} \times Y \times A \longrightarrow X \times Y \times Z \\
& i \times i \times 1_{Z}:\{x\} \times\{y\} \times Z \longrightarrow X \times Y \times Z
\end{aligned}
$$

where $f \in M\left(A^{\prime}, Y\right), g \in M(A, Z), A, A^{\prime} \in \mathscr{A}, x \in X, y \in Y$.

(ii) If $A, A^{\prime} \in \mathscr{A}, f \in M(A, Y), g \in M\left(A^{\prime}, Z\right)$ then $A^{\prime \prime}=A \times A^{\prime} \in$ $\mathscr{A}$ and

$$
1 \times f \times g: X \times A \times A^{\prime} \longrightarrow X \times Y \times Z
$$

can be expressed in the form

$$
1 \times\left(f \operatorname{proj}_{A}, g \operatorname{proj}_{A}\right): X \times A^{\prime \prime} \longrightarrow X \times Y \times Z ;
$$

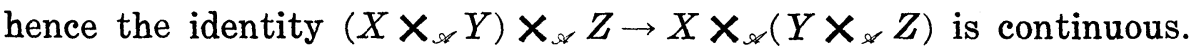

(iii) It follows from Lemma 0.2 that there is an identification map $p: C=\bigcup_{\lambda \in A} C_{\lambda} \rightarrow X$, where each $C_{\lambda}$ is compact Hausdorff. Now $A \times A^{\prime}$ is an $a$-space so there is an identification $q: A^{\prime \prime}=\bigcup_{\mu \in M} A_{\mu}^{\prime \prime} \rightarrow$ $A \times A^{\prime}$ and the composite

$$
C \times A_{\mu}^{\prime \prime} \underset{1 \times q}{\longrightarrow} C \times A \times A^{\prime} \underset{p \times 1_{A} \times 1_{A^{\prime}}}{\longrightarrow} X \times A \times A^{\prime}
$$

is also an identification; the last fact following by the argument used at the beginning of (ii). The composites

$$
C_{\alpha} \times A_{\mu}^{\prime \prime} \longrightarrow X \times A \times A^{\prime} \underset{1 \times f \times g}{\longrightarrow}\left(X \times{ }_{\star} Y\right) \times_{\curvearrowleft} Z
$$

factor through the maps

$$
1 \times(f, g): X \times A \longrightarrow X \times \mathscr{A}(Y \times, Z)
$$

and the result follows.

2. Exponential laws and monoidal closed structures for Top.

Proposition 2.1. (Proper condition, compare with [1, Theorem 4.21].) If the spaces in . are compact and $f: X \times, Y \rightarrow Z$ is continuous then $f(x, y)=f^{\prime}(x)(y)$ determines a continuous function $f^{\prime}: X \rightarrow M_{\Re}(Y, Z)$.

Proof. For each $x \in X, f^{\prime}(x)$ is simply the composite

$$
Y \cong\{x\} \times Y \underset{\text { inclusion }}{\longrightarrow} X \times \times_{\mathscr{A}}^{\longrightarrow} \underset{f}{\longrightarrow} Z,
$$

the inclusion is continuous from the definition of $X \times{ }_{\star} Y$, and hence $f^{\prime}$ is a well defined function. 
Now let $W=W(g, U)$ be a subbasic open set for $M_{\mathscr{A}}(Y, Z)$, where $g: A \rightarrow Y$. If $k=f(1 \times g): X \times A \rightarrow Z$ then $k$ is continuous and $k^{-1}(U)$ is open in $X \times A$. Given $x \in\left(f^{\prime}\right)^{-1}(W)$ it is clear that $\{x\} \times A \subseteq k^{-1}(U)$. Now $A$ is compact and so there exists an open set $V \subseteq X$ such that $x \in V$ and $V \times A \subseteq k^{-1}(U)$ [14, Theorem 5.12]. This implies that $x \in V \subseteq\left(f^{\prime}\right)^{-1}(W)$ and so $\left(f^{\prime}\right)^{-1}(W)$ is open.

Definition 2.2. The class $\mathscr{A}$ of spaces will be said to be a regular class if for any element $k$ of a set $A$ in $\mathscr{A}$, any neighborhood of $k$ contains a closed neighborhood $C$ for which there is a surjective map $r: B \rightarrow C$ with $B \in \mathscr{A}$. We notice that the individual spaces in $\mathscr{A}$ must then, of course, themselves be regular.

Proposition 2.3 (Admissible condition, compare with [1, Theorem 4.7].) If . $\mathscr{A}$ is a regular class of spaces and $f^{\prime}: X \rightarrow M_{\mathscr{N}}(Y, Z)$ is continuous, then so is the associated function $f: X \times \propto Y \rightarrow Z$.

Proof. We show that the evaluation map,

$$
e: M_{\mathscr{A}}(Y, Z) \times_{\mathscr{A}} Y \longrightarrow Z, \quad e(g, y)=g(y),
$$

is continuous and the result will follow since

$$
f=e\left(f^{\prime} \times 1_{Y}\right) .
$$

Using the universal property of final topologies and noticing that

$$
e\left(i \times 1_{Y}\right):\{h\} \times Y \longrightarrow Z
$$

is clearly continuous for all $h \in M_{\curvearrowright}(Y, Z)$, we just have to prove that

$$
\begin{aligned}
& e_{k}=e(1 \times k): M_{\Im}(Y, Z) \times A \longrightarrow Z, e_{k}(g, a)=g k(a) \text { where } \\
& k \in M(A, Y), \text { is continuous } .
\end{aligned}
$$

Let $U$ be an open subset of $Z$ and $(g, a) \in e_{k}^{-1}(U)$. Then the regularity of $\mathscr{A}$ ensures that there is an open set $V$ and a closed set $C$ such that

$$
a \in V \subseteq C \leqq k^{-1} g^{-1}(U) \quad \text { with } \quad W(k \mid C, U)=W(k r, U),
$$

for a suitable choice of $r$. Hence $W(k \mid C, U)$ is a subbasic open set for $M_{\mathscr{N}}(Y, Z)$ and $g(a) \in(W(k \mid C, U) \times V)^{\text {open }} \subseteq e_{k}^{-1}(U)$ ensures that $e_{k}^{-1}(U)$ is open and that $e_{k}$ is continuous.

THEOREM 2.4 (Exponential law). (i) Given that $X, Y, Z$ are spaces and $\mathscr{A}$ is a regular class of compact spaces. The function 


$$
\begin{aligned}
& \theta: M_{\mathscr{N}}\left(X, M_{\mathscr{N}}(Y, Z)\right) \longrightarrow M_{\mathscr{M}}\left(X \times_{\circlearrowleft} Y, Z\right), \\
& \theta\left(f^{\prime}\right)=f, f(x, y)=f^{\prime}(x)(y), x \in X, y \in Y,
\end{aligned}
$$

is a continuous bijection.

(ii) If, in addition, $\mathscr{A}$ is a class of Hausdorff spaces closed under the formation of cartesian products then $\theta$ is a homeomorphism.

Proof. (i) It follows from 1.4(i) and the continuity of the evaluation maps $e$ (see the proof of 2.3) that the composite

$$
\begin{aligned}
& M_{\mathscr{A}}\left(X, M_{\mathscr{N}}(Y, Z)\right) \mathrm{X}_{\mathscr{N}}\left(X \times_{\mathscr{A}} Y\right) \underset{1}{\longrightarrow}\left(M_{\mathscr{N}}\left(X, M_{\mathscr{A}}(Y, Z)\right) \mathrm{X}_{\mathscr{A}} X\right) \\
& \mathrm{X}_{\mathscr{S}} Y \underset{e \times 1}{\longrightarrow} M_{\mathscr{A}}(Y, Z) \times \underset{e}{\longrightarrow} Z
\end{aligned}
$$

is continuous; the result follows from 2.1.

(ii) This follows from 1.4 (ii), 2.1, 2.3 and [15, Theorem 10].

DEFINITION 2.5. A monoidal closed structure for Top will mean a 4-tuple (ToP, $\left\{{ }^{*}\right\}, X_{\mathscr{N}}, M_{\mathscr{A}}(-,-)$ ), where $\left\{{ }^{*}\right\}$ is a singleton space and $X_{s}$ and $M_{s}(-,-)$ are the product and function space bifunctors associated with some class $\mathscr{A}$, subject to the following axioms:

(i) there is an exponential homeomorphism as in Theorem 2.4(ii);

(ii) there is a natural homeomorphism

$$
\phi: X \longrightarrow M_{\sim}\left(\left\{^{*}\right\}, X\right), \phi(x)\left(^{*}\right)=x, x \in X .
$$

It follows from [10, p. 495, Theorem 5.10] that any such structure can be completed to determine a monoidal closed category $[10, p$. 475].

THEOREM 2.6. If . is a regular class of compact Hausdorff spaces containing at least one nonempty space and closed under the formation of Cartesian products then the product $X \times Y$ and the function space $M(Y, Z)$ determine a monoidal closed structure for ToP.

Proof. Condition (i) follows from 2.4(ii); condition (ii) either from the definition of $M_{\Im}(Y, Z)$ or 1.2 and the first sentence of [10, p. 480].

REMARK 2.10. If $X, Y$ are Hausdorff (or $T_{1}$ or regular) then $X \times, Y$ is Hausdorff (or $T_{1}$ or regular respectively). This follows from the continuity of the identity $X \times \mathscr{A} \rightarrow X \times Y$. If $Z$ is Hausdorff (or $T_{1}$ or regular) then $M_{\mathscr{N}}(Y, Z)$ is Hausdorff (or $T_{1}$ or regular 
respectively), the regular case requiring that $\mathscr{A}$ is a class of compact spaces. The proofs are similar to the analogous standard results for the compact-open topology. Hence Theorem 2.5 also gives sufficient conditions for the existence of monoidal closed structures on HAUS (and on the categories of $T_{1}$-spaces and regular spaces).

3. Exponential law relating cartesian products to function spaces with the $\mathscr{A}$-open topology.

THEOREM 3.1. Let $\mathscr{A}$ be a regular class of compact spaces and $X, Y$ and $Z$ be given spaces.

(i) The function $\theta: M_{\mathscr{N}}\left(X, M_{\mathscr{N}}(Y, Z)\right) \rightarrow M_{\mathscr{A}}(X \times Y, Z)$ is a continuous bijection if

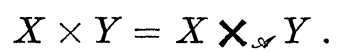

(ii) If, in addition, $\mathscr{A}$ consists of Hausdorff spaces and is closed under the formation of cartesian products then $\theta$ is a homeomorphism if and only if $X \times Y=X \times{ }_{\leftrightarrow} Y$.

Proof. This follows easily from Theorem 2.4.

Hence the problem of determining conditions under which there is a valid exponential law relating $X \times Y$ with $M_{\mathscr{A}}(Y, Z)$ reduces to finding conditions under which $X \times Y=X \times Y Y$. Criteria of this type are given by the next result.

Definition 3.2. The space $Y$ is said to be locally . $\mathscr{\text { if }}$ each point $y \in Y$ has a neighborhood $A_{y}$ homeomorphic to a space in $\mathscr{A}$.

Proposition 3.3. Given spaces $X$ and $Y$ and a class of spaces $\mathscr{A}$, if either (i) $Y$ is locally $\mathscr{A}$ or (ii) $X \times Y$ is an a-space then $X \times Y=X \times_{\mathscr{A}} Y$.

Proof. We have to show that $1: X \times Y \rightarrow X \times{ }_{\mathscr{A}} Y$ is continuous.

(i) We notice that for each choice of $y$ the composite

$$
X \times V_{y} \underset{i}{\longrightarrow} X \times A_{y} \underset{1 \times i}{\longrightarrow} X \times \times_{\mathscr{A}} Y,
$$

is continuous where $x \in V_{y} \subseteq A_{y}$ and $V_{y}$ is open in $Y$. If $U$ is open in $X \times_{\nsim} Y$ it follows that $U \cap\left(X \times V_{y}\right)$ is open in $X \times V_{y}$, and therefore in $X \times Y$. Hence

$$
U=\bigcup_{y \in Y} U \cap\left(X \times V_{y}\right) \text { is open in } X \times Y .
$$


(ii) 1: $a(X \times Y) \rightarrow X \times{ }_{\mathscr{A}} Y$ is continuous, and the result follows since $X \times Y=a(X \times Y)$.

4. Cartesian closed categories of topological spaces. The reader will recall that a category of topological spaces is Cartesian closed $[10$, p. 551] if it includes a singleton space and is equipped with categorical product and function space bifunctors related by an exponential law. It is well known (see e.g., [8, Theorem 3.1], [19], [23, §3] and [25, Theorem 3.3]) that with suitable restrictions on . A the category of $a$-spaces is cartesian closed. We prove below that these "cartesian closed" exponential laws are simply the restriction of our exponential law (Theorem 2.4) to categories of a-spaces.

Our first result is essentially a slight generalization of [23, Corollary $3.3(\mathrm{~b})]$.

LeMma 4.1. Let $\mathscr{L}$ be a class of locally compact Housdorff spaces satisfying the condition that $A \times B$ is an a-space for any choice of $A, B \in \mathscr{A}$. If $X$ is an a-space and $Y \in \mathscr{A}$ then $X \times Y=$ $a(X \times Y)$.

Proof. Given that $Z$ is a space and $f: X \times Y \rightarrow Z$ is a function. The continuity of $f$ is equivalent to the continuity of the corresponding $f^{\prime}: X \rightarrow \mathrm{M}_{\mathrm{cot}}(Y, Z)$, and hence to the continuity of all functions $f^{\prime} g: A \rightarrow \mathrm{M}_{\mathrm{cot}}(Y, Z)$ where $A \in \mathscr{A}$ and $g \in M(A, Y)$. Now this last condition is equivalent to the continuity of all $f\left(g \times 1_{Y}\right)$ : $A \times Y \rightarrow Z$ and so $A \times Y$ has the final topology relative to all maps $g \times 1_{Y}: A \times Y \rightarrow X \times Y$. We notice that maps $(g, h): A \rightarrow X \times Y$ factor through $g \times 1_{Y}$; it follows by the composition rule for final topologies $[5$, p. 96] that $X \times Y$ is an $a$-space.

REMARK 4.2. The identities $a(X \times Y) \rightarrow X \times{ }_{\mathscr{A}} Y \rightarrow X \times Y$ are continuous and so, under the assumptions of 4.1, $a(X \times Y)=X \times{ }_{*}=$ $X \times Y$. The condition for $a(X \times Y)=X \times{ }_{\mathscr{S}} Y$ is generalized in the next result.

Proposition 4.3. Given that $\mathscr{A}$ is a class of locally compact Hausdorff spaces satisfying the condition that $A \times B$ is an a-space for any $A, B \in \mathscr{A}$. If $X$ and $Y$ are a-spaces then $X \times, Y=a(X \times Y)$.

Proof. Given that $A \in \mathscr{A}, X \times A$ is an $a$-space [23, Corollary 3.3]; it follows that $X \times, Y$ has the final topology with respect to all maps

$$
(f, g): A^{\prime} \longrightarrow X \times Y, i \times h:\{x\} \times A^{\prime} \longrightarrow X \times Y,
$$


where $A^{\prime} \in \mathscr{A}, f \in M\left(A^{\prime}, X\right), g \in M\left(A^{\prime}, Y\right), h \in M(A, Y), i:\{x\} \rightarrow X$ is the inclusion and $x \in X$. Hence $X \times_{\mathscr{N}} Y$ has the final topology relative to all incoming maps from spaces in $\mathscr{A}$.

THEOREM 4.4. If $\mathscr{A}$ is a regular class of compact Hausdorff spaces satisfying the condition that $A \times B$ is an a-space whenever $A, B \in \mathscr{A}$, then the category of a-spaces with product $a(X \times Y)$ and function space aM $M_{\mathscr{r}}(Y, Z)$ is a cartesian closed category.

Proof.

$$
\begin{aligned}
M\left(X, a M_{\mathscr{A}}(Y, Z)\right. & =M\left(X, M_{\mathscr{A}}(Y, Z)\right)[23, \text { Corollary 1.4] } \\
& =M(X \times \mathscr{Y}, Z) 2.4(\mathrm{i}) \\
& =M(a(X \times Y), Z) 4.3
\end{aligned}
$$

and the result follows.

REMARK 4.5. The other approaches mentioned at the beginning of this section give slightly stronger results than our 4.4; we can in each case delete the regular class requirement, the last two

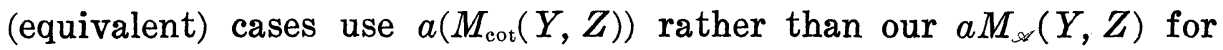
their function spaces. Our version seems, however, to include all of the important examples. Also it follows that given spaces $Y$ and $Z$ and a class $\mathscr{A}$ satisfying the conditions of Theorem 4.4 then

$$
a M_{\mathscr{A}}(Y, Z)=a M_{\mathrm{cot}}(Y, Z) .
$$

5. Locally $\mathscr{A}$ spaces and $a$-spaces.

Proposition 5.1. If the space $Y$ is locally then $Y$ is an aspace.

Proof. If $U$ is open in $a Y$ then $U \cap A_{y}$ is open in $A_{y}$ for all $y \in Y$, where $\left\{A_{y}\right\}_{y \in Y}$ is the cover of $Y$ with open subcover $\left\{U_{y}\right\}_{y \in Y}$ whose existence is implied by the data. It follows that $U \cap U_{y}$ is open in $U_{y}$ for all $y \in Y$; hence $U \cap U_{y}$ is open in $Y$ and $U=\bigcup_{y \in Y} U \cap U_{y}$ is open in $Y$. So the identity function $Y \rightarrow a Y$ is continuous and hence is a homeomorphism.

TheORem 5.2. Let $\mathscr{A}$ be any class of spaces containing at least one nonempty space and $\mathscr{C}$ be a class of a-spaces containing all topological sums of spaces in $\mathscr{A}$. The class of all a-spaces is then the class of all quotients of spaces in $\mathscr{C}$. In particular the class of all a-spaces can be characterized as either (i) the class of all quotients of topological sums of spaces in $\mathscr{A}$ or (ii) the class of all quotients of locally spaces. 
Proof. If $X$ is an $a$-space then it is a quotient of a topological sum of spaces in $\mathscr{A}$ (see Lemma 0.2 ) and hence a quotient of spaces in $\mathscr{C}$. Conversely any quotient of a space in $\mathscr{C}$ is a quotient of an $a$-space, and hence is itself an $a$-space [23, Corollary 2.2].

6. First example: the sequential case.

(i) We will take $\mathscr{C}=\mathscr{C} \mathscr{S}$, the class whose only element is $N_{\infty}$, the one point compactification of the natural numbers with the discrete topology. Then $M_{\text {es }}(Y, Z)$ carries the $\mathscr{C} \mathscr{S}$-open topology (convergent sequence-open topology) of [12], [13]. Now $\mathscr{C} \mathscr{S}$ is a regular class consisting of one compact Hausdorff space and it follows from 2.4(i) that there is a continuous bijection:

$$
M_{\mathscr{B S}}\left(X, M_{\mathscr{B S}}(Y, Z)\right) \longrightarrow M_{\mathscr{B S}}\left(X \times_{\mathscr{B S}} Y, Z\right) \text {. }
$$

Now $\mathscr{C} \mathscr{S}$ is not closed under the formation of cartesian products, so it is not clear if the $\mathscr{C} \mathscr{S}$-product is associative, if $\theta$ is a homeomorphism or if this determines a monoidal closed structure on ToP.

The associated category of $a$-spaces is the category of sequential spaces.

It follows from 3.1 and 3.3 that if $X$ and $Y$ are spaces whose product is a sequential space then

$$
M_{\mathscr{B} \mathscr{S}}\left(X, M_{\mathscr{B S}}(Y, Z)\right) \cong M_{\mathscr{B S}}(X \times Y, Z) .
$$

Varıous characterizations of the category of sequential spaces follow from our 5.2, i.e., it is:

(a) the category of metrizable spaces and their quotients,

(b) the category of first countable spaces and their quotients,

(c) the category of direct sums of copies of $N_{\infty}$ and their quotients,

(d) the category of all locally compact metrizable spaces and their quotients,

(e) the category of all direct sums of compact metrizable spaces and their quotients,

and

(f) the category of all $M$-spaces [16] and their quotients ((a), (b), (c) are given in [11]).

A detailed discussion of most of the points raised in this example is given in [21].

Our sequential exponential laws should not be confused with the analagous results for $L^{*}$-spaces $\left[18\right.$, p. 200, Theorem $\left.3^{\prime}\right]$ and "convergences" [21, Theorem 2.5]. These "objects", although they can be generated by $N_{\infty}$, are not topological spaces; the relationship between such exponential laws and those for sequential spaces is 
discussed in [21, Chapter III].

\section{Convenient categories.}

Definition 7.1. Given a class $\mathscr{A}$, the associated category of $a$-spaces is said to be a convenient topological category if:

(i) it is cartesian closed, and

(ii) it contains the underlying topological spaces of all $C W$ complexes, all differentiable manifolds and all metric spaces.

The category is then "closed under certain standard operations" (it has limits and colimits, subspaces and quotients), (i) ensures that it is small enough to satisfy "certain reasonable identities" and (ii) that is large enough to contain (just about) "all of the particular spaces arising in practice"; it follows that it is convenient in the sense of Steenrod [20]. For more details of these arguments the reader is referred to [23, 2.1 and 3.7].

REMARK 7.2. Some limits of spaces (e.g., any cartesian product of $C W$-complexes which fails to be a $C W$-complex) and some function spaces (e.g., with the compact-open topology) may be outside a convenient category. We can, however, replace any such spaces by their images under the appropriate functor $a$. The fact that a convenient category includes the standard spheres $S^{n}$ and cylinders $S^{n} \times I$ ensures that the identity $a X \rightarrow X$ is a weak homotopy equivalence and induces isomorphisms of homotopy, homology and cohomology groups [23, 1.2(h) and 5.3(d)].

Proposition 7.3. Given that $\mathscr{A}$ generates a cartesian closed category of a-spaces. The following conditions are equivalent

(i) the category of a-spaces is convenient,

(ii) $N_{\infty}$ is an a-space,

(iii) all sequential spaces are a-spaces.

Proof. The condition (i) $\Rightarrow$ (ii) because $N_{\infty}$ is metrizable, (ii) $\Rightarrow$ (iii) follows from (c) above and [23, p. 548] and (iii) $\Rightarrow$ (i) is immediate.

THEOREM 7.4. The category of sequential spaces is the smallest convenient topological category.

Proof. This is immediate from 7.3(iii).

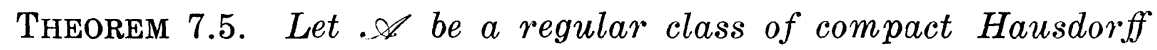
spaces satisfying the conditions that:

(i ) $N_{\infty}$ is an a-space, 
(ii) $A \times B$ is an a-space whenever $A, B \in \mathscr{A}$. Then the category of a-spaces is a convenient topological category.

Proof. This follows from 6.1 (ii) and 4.4.

REMaRK 7.6. (a) Condition (i) is satisfied if $N_{\infty} \in \mathscr{L}[23,1.2(\mathrm{~d})]$. (b) If we use a different function space, i.e., $a M_{\text {cot }}(Y, Z)$ rather than $a M_{M}(Y, Z)$, then the "regular class" condition can be deleted from 7.5 (see Remark 4.5).

8. Further examples. The sequential example has been considered in $\S 6$. Each of the examples (ii), (iii), (iv), (v), (vi), (vii) and (viii) below is determined by a regular class of compact Hausdorff spaces, hence each satisfies an exponential law as in 2.4(i). These cases all involve classes closed under the formation of cartesian products and hence determine exponential homeomorphisms; all but (ii) generate monoidal closed structures for Top (see 2.6). Example (v) is the monoidal closed structure for the category of all Hausdorff spaces associated with the compact-open topology and referred to in the introduction.

(ii) Let us take $\mathscr{\mathscr { C }}=\mathscr{E}$, the empty class of spaces; then $M_{\&}(Y, Z)$ has the indiscrete topology and $X \times_{\mathscr{E}} Y$ has the final topology with respect to all inclusions

$$
\{x\} \times Y \longrightarrow X \times Y, \quad x \in X
$$

(and not the discrete topology as stated in [24, p. 277] (. It is easily seen that this condition ensures that a given function $f: X \times Y \rightarrow Z$ is continuous if and only if the associated $f^{\prime}: X \rightarrow M(Y, Z)$ is well defined, the continuity of $f^{\prime}$ being immediate $X X^{*}\left\{^{*}\right\}$ is discrete and so the structure is not monoidal closed.

The idea of being locally $\mathscr{E}$ (i.e. locally empty) is self contradictory! The associated cartesian closed category is the category of all discrete spaces; it is not convenient, however, as it does not contain many of "the particular spaces arising in practice."

(iii) We now take $\mathscr{A}=\mathscr{F} \mathscr{D}$, the class of all finite discrete spaces; then $M_{r}(Y, Z)$ has the topology of pointwise convergence. It is known [4, Remark 1.15], [17, Theorem 1.5], [24, p. 277] and [25, p. 226-227] that this determines an exponential law and hence a monoidal closed structure on ToP.

If $Y$ is discrete (=locally $\mathscr{F} \mathscr{D}$ ) then $X \times{ }_{\mathscr{O}} Y=X \times Y$ and

$$
\theta: M_{\mathscr{F} \mathscr{C}}\left(X, M_{\mathscr{T} \mathscr{Z}}(Y, Z)\right) \longrightarrow M_{\mathscr{S} \mathscr{Z}}(X \times Y, Z)
$$

is a homeomorphism. The corresponding cartesian closed category is, as in example (i), the category of discrete spaces. 
(iv) Let us take $\mathscr{C}=\mathscr{C} \mathscr{H}$, the class of all compact Hausdorff spaces. Then ToP with the exponential law.

$$
M_{\mathscr{E} \mathscr{C}}\left(X, M_{\mathscr{B} \mathscr{F}}(Y, Z)\right) \cong M_{\mathscr{E} \mathscr{P}}\left(X \mathrm{X}_{\mathscr{B} \mathscr{X}} Y, Z\right)
$$

is monoidal closed.

It follows from 3.1 and 3.3 that if either (i) $Y$ is locally compact and Hausdorff or if (ii) $X \times Y$ is a $k$-space then

$$
M_{\mathscr{C} \mathscr{E}}\left(X, M_{\mathscr{\mathscr { X }}}(Y, Z)\right) \cong M_{\mathscr{E} \mathscr{X}}(X \times Y, Z) .
$$

The associated convenient category is that of $k$-spaces [7], [2, p. 276] and [23, p. 558]. It is well known (and follows from 5.2) that this is the category of all locally compact Hausdorff spaces and their quotients.

(v) If $X$ and $Y$ are Hausdorff then $M_{\mathscr{C} \mathscr{C}}(Y, Z)$ has the compactopen topology $\left(=M_{\text {cot }}(Y, Z)\right)\left[7\right.$, p. 23] and $X X_{\mathscr{8} X} Y$ coincides with the product $X \times_{s} Y$ of $[3$, p. 309-310], we then have the exponential law

$$
M_{\text {cot }}\left(X, M_{\text {cot }}(Y, Z)\right) \cong M_{\text {cot }}\left(X \times_{s} Y, Z\right)
$$

(=[4, Theorem 1.6]). Hence taking $\mathscr{A}=\mathscr{C} \mathscr{H}$ and working with the compact-open topology and HAUs we obtain a monoidal closed structure on that category.

The last homeomorphism of example (iv) then reduces to the following standard theorem ( $=[9$, p. 265, Theorem 3]): If $X, Y$ are Hausdorff and either (i) $Y$ is locally compact or (ii) $X \times Y$ is compactly generated, then

$$
M_{\text {cot }}\left(X, M_{\text {cot }}(Y, Z)\right) \cong M_{\text {cot }}(X \times Y, Z) .
$$

The associated convenient category is that of compactly-generated spaces.

(vi) We will now take $\mathscr{A}=\mathscr{C} \mathscr{M}$ the class of all compact metrizable spaces.

It follows from 3.1 and 3.3 that: If either (i) $Y$ is locally compactmetrizable, or (ii) $X \times Y$ is a sequential space (e.g., $X, Y$ first-countable or $X, Y$ spaces whose product is a $C W$-complex) then

$$
M_{\mathscr{C} M}\left(X, M_{\mathscr{C}}(Y, Z)\right) \cong M_{\mathscr{C}}(X \times Y, Z) .
$$

The corresponding convenient category is that of sequential spaces [25, p. 236-237]; for more details see example (i) above.

(vii) Let us now take $\mathscr{A}=\mathscr{C} \mathscr{B}$ the class of all cubes $I^{n}$ ( $I$ denotes the unit interval, $n>0$ and finite). The associated cartesian closed category has been discussed in [8, p. 6] and [25, p. 236-237].

It follows from 5.2 that is can be characterized as either 
(a) the category of all underlying spaces of $C W$-complexes and their quotients $[8, \mathrm{p} .6]$,

(b) the category of all locally Euclidean spaces and their quotients,

(c) the category of all topological sums of cubes $I^{n}$ and the quotients of such sums, and

(d) the category of all topological sums of copies of the unit interval $I$ and the quotients of such sums [25, p. 237] (the proof of (d) involves (c), the fact that each $I^{n}$ has the final topology with respect to all incoming maps from $I$ and the composition rule $[5, \mathrm{p}$. 96] for final topologies).

It follows from 3.3 and (a) above that: if either (i) $Y$ is locally Euclidean or (ii) $X$ and $Y$ are spaces whose product is the underlying space of a $C W$-complex then

$$
M_{\mathscr{B} \mathscr{B}}\left(X, M_{\mathscr{C} \mathscr{B}}(Y, Z) \cong M_{\mathscr{E} \mathscr{B}}(X \times Y, Z) .\right.
$$

The associated, cartesian closed category, although adequate for many purposes in Algebraic Topology, is not a convenient topological category sense. The reason is that it does not contain all metric spaces; in particular it does not contain $N_{\infty}$, for it is easily seen that the corresponding $a N_{\infty}$ carries the discrete topology.

(vii) Finally we take $\mathscr{A}=\mathscr{C} \mathscr{C} \mathscr{C}$, the set of compact Hausdorff spaces whose underlying set is countable. A monoidal closed structure for ToP and associated convenient category are determined. Now countable compact Hausdorff spaces are metrizable, so

$$
\left\{N_{\infty}\right\} \subset \mathscr{C} \mathscr{H} \mathscr{C} \subset \mathscr{C} \mathscr{M}
$$

and the associated convenient category is that of sequential spaces [25, p. 237].

REMARK 8.1. Many other classes of spaces $\mathscr{A}$ determine monoidal closed structures for TOP and cartesian closed (and often convenient) categories of $a$-spaces. Assume for example that $P$ is any property of topological spaces that is productive [14, p. 133] and closed hereditary, the last condition meaning that if a space satisfies $P$ then all of its closed subspaces satisfy $P$. It follows that if

$\mathscr{A}=$ the class of all compact Hausdorff spaces satisfying $P$

contains a nonempty space then it generates a monoidal closed structure for ToP and a cartesian closed category of $a$-spaces. The reader might also consider the results of taking $\mathscr{A}=\{I\}$ or $. \mathscr{A}=$ $\left\{N_{\infty}^{n} \mid n=a\right.$ finite integer $\}$.

It is not, however, clear to the authors that any of the modified 
products, function spaces $M_{\mathscr{r}}(Y, Z)$ and categories of $a$-spaces generated are distinct from examples (i) ... (viii) above (compare with $\S 9)$.

9. Comparison of function space topologies. It is clear that if $\mathscr{A}_{1}, \mathscr{A}_{2}$ are classes of spaces and $\mathscr{A}_{1} \subseteq \mathscr{A}_{2}$ then the $\mathscr{A}_{1}$-open topology is contained in the $\mathscr{A}_{2}$-open topology; hence the following identity functions are continuous:

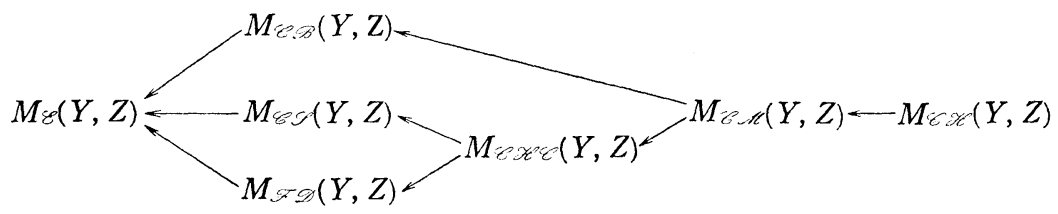

The $\mathscr{E}$-open and $\mathscr{F} \mathscr{D}$-open topologies are the indiscrete topology and the topology of pointwise convergence respectively; furthermore the latter is completely independent of the topology on $Y$, so these two are in general distinct from the others. It is shown in $[13, p$. 110] that the C.SP-open topology is in general distinct from the compact-open topology; the same argument shows that both the $\mathscr{C} \mathscr{S}$-open and $\mathscr{C} \mathscr{Y C} \mathscr{C}$-open topologies differ from all of the $\mathscr{C} \mathscr{P}$ open, $\mathscr{C} \cdot \mathscr{C l}$-open and $\mathscr{C} \mathscr{B}$-open topologies. The essential point here being that one cannot cover the unit interval with union of a finite countable sets.

Lemma 9.1. Let $\mathscr{\text { A, }} \mathscr{\mathscr { A }}^{\prime}$ be regular classes of compact Hausdorff spaces closed under the formation of cartesian products and $Y$ be a given space. Then $M_{\mathscr{r}}(Y, Z)=M_{r}(Y, Z)$ for all spaces $Z$ if and only if $X \times_{\mathscr{A}} Y=X \times_{\mathscr{A}}, Y$ for all spaces $X$.

Proof. This follows from Theorem 2.4 and the uniqueness of adjoints.

Proposition 9.2. $M_{\mathscr{E} \mathscr{F}}(Y, Z) \neq M_{\mathscr{E} \mathfrak{M}}(Y, Z)$, in general

Proof. It is sufficient, by the previous lemma, to show

$$
N_{\infty} \times \ldots N_{\infty} \neq N_{\infty} \mathbf{X}_{\mathscr{C} \mathscr{B}} N_{\infty} .
$$

Consider the set:

$$
D=\left\{(n, n) \mid n \in N_{\infty}\right\} \subset N_{\infty}^{2} .
$$

$N_{\infty} \times_{\&}, N_{\infty}=N_{\infty} \times N_{\infty}$ (by Lemma 3.3) and $D$ is not closed in that space. On the other hand all maps from $I^{n}$ into $N_{\infty}$ are constant and 
so $N_{\infty} \mathrm{X}_{\mathscr{C} . \infty} N_{\infty}$ has the final topology with respect to all inclusions

$$
\{x\} \times N_{\infty} \longrightarrow N_{\infty} \times N_{\infty}, N_{\infty} \times\{y\} \longrightarrow N_{\infty} \times N_{\infty}, x, y \in N_{\infty} .
$$

Hence $D$ is closed in $N_{\infty} X_{\mathscr{B} \mathscr{B}} N_{\infty}[5$, p. 93,4.22(b)] and the result follows.

Hence we have proved the following result.

THEOREM 9.3. There are at least five distinct set-open function space topologies on $M(Y, Z)$ (indiscrete, pointwise-convergence, $\mathscr{C} \mathscr{H}$ open, $\mathscr{C} \mathscr{B}$-open and $\mathscr{C} \mathscr{H} \mathscr{C}$-open) that have associated exponential homeomorphisms in ToP.

We have not been able to determine whether:

(i) $M_{\mathscr{B} \mathscr{E}}(Y, Z)$ and $M_{\mathscr{8}}(Y, Z)$ are in general distinct;

and

(ii) $M_{\mathscr{B} \mathscr{C}}(Y, Z)$ and $M_{\mathscr{B} \mathscr{X}}(Y, Z)$ are in general distinct.

Question (i) is related to the problem of whether $M_{\mathscr{E S} S}(Y, Z)$ determines a monoidal closed structure on ToP; question (ii) is of some interest because $\mathscr{C} \mathscr{H}$ and $\mathscr{C} \mathscr{C}$ give rise to different convenient categories.

Proposition 9.4. Given spaces $Y, Z$.

(i) If $Y$ is a discrete space then

$$
\begin{aligned}
M_{\mathscr{S} \mathscr{T}}(Y, Z) & =M_{\mathscr{B} \mathscr{C}}(Y, Z)=M_{\mathscr{B} \mathscr{A}}(Y, Z)=M_{\mathscr{B} \mathscr{B}}(Y, Z) \\
& =M_{\mathscr{B} \mathscr{A}}(Y, Z)=M_{\mathscr{B} \mathscr{A}}(Y, Z)=M_{\mathrm{cot}}(Y, Z) .
\end{aligned}
$$

(ii) If $Y$ is locally Euclidean

$$
M_{\mathscr{\mathscr { C }} \mathscr{C}}(Y, Z)=M_{\mathscr{E} \mathscr{H}}(Y, Z)=M_{\mathscr{E} \mathscr{B}}(Y, Z) .
$$

(iii) If $Y$ is metrizable

$$
M_{\mathscr{C} \mathscr{E}}(Y, Z)=M_{\mathscr{Q} M}(Y, Z)=M_{\mathrm{cot}}(Y, Z) .
$$

Proof. (i) For each choice of the subsets $f(A)$ of $Y$ are compact, and hence are either the finite subsets or the singleton subsets; hence the function space topologies coincide.

(ii) We have, for all spaces $X$

$$
X \times_{\mathscr{B} \mathscr{C}} Y=X \times_{\mathscr{B} M} Y=X \times_{\mathscr{B} \mathscr{B}} Y=X \times Y
$$

(by 3,3 ); the result follows by 9.1 .

(iii) This is immediate from the definitions involved.

ACKNOWLEDGMent. Georg Grene has pointed out the necessity 
of excluding the case $\mathscr{A}=\mathscr{E}$ from 1.2 and 2.6 , hence $\mathscr{E}$ does not therefore determine a monoidal closed structure on ToP.

\section{REFERENCES}

1. R. Arens and J. Dugundji, Topologies for function spaces, Pacific J. Math., 1 (1951), 5-31.

2. P. Booth, The section problem and the lifting problem, Math. Z., 121 (1971), 273-287.

3. R. Brown, Ten topologies for $X \times Y$, Quart J. Math., (2) 14 (1963), 303-319.

4. —, Function spaces and product Topologies, Quart J. Math., (2) 15 (1964), 238-250.

5. — Elements of Modern Topology, McGraw-Hill, London, 1968.

6. - On sequentially proper maps and a sequential compactification, J. London Math. Soc., (2) 7 (1973), 1-8.

7. A. Clark, Quasi-Topology and Compactly Generated Spaces, Mimeographed Notes, Brown University (about 1967), (unpublished).

8. B. Day, A reflection theorem for closed categories, J. Pure and Appl. Algebra, 2 (1972), 1-11.

9. J. Dugundji, Topology, Allyn and Bacon, 1966.

10. S. Eilenberg and G. M. Kelly, Closed Categories, Proc. Conf. on Categorical Algebra, La Jolla, 1965, Springer-Verlag, Berlin, 1969, 541-552.

11. S. P. Franklin, Spaces in which sequences suffice, Fund. Math., 57 (1965), 107-115.

12. J. Guthrie, On some generalizations of metric spaces, Ph. D. Dissertation, Texas Christian University, 1969.

13. - A characterization of $\boldsymbol{\aleph}_{0}$-spaces, General Topology and its Appl., 1 (1971), $105-110$.

14. J. L. Kelley, General Topology, Van Nostrand, Princeton, 1955.

15. G. M. Kolly, Tensor products in categories, J. Algebra, 2 (1965), 15-37.

16. D. M. Hyman, A category slightly larger than the metric and $C W$-categories, Michigan Math. J., 15 (1968), 193-214.

17. C. J. Knight, W. Moran and J. S. Pym, The topologies of separate continuity I, Proc. Camb. Phil. Soc., 68 (1970), 663-671.

18. K. Kuratowski, Topology, Vol. I, Academic Press, New York and London, 1966.

19. L. D. Nel, Cartesian closed coreflective hulls, Quaestiones Mathematicae, 2 (1977), 269-283.

20. N. E. Steenrod, A convenient category of topological spaces, Michigan Math. J., 14 (1967), 133-152.

21. J. Tillotson, The convenient category of sequential spaces, Masters thesis, Memorial University of Newfoundland, 1978.

22. R. Vazquez, Espacios funcionales y funtores adjuntos, An. Inst. Math. Univ. Nac. Autónoma México, 6 (1966), 7-46.

23. R. M. Vogt, Convenient categories of topological spaces for homotopy theory, Archiv der Math., 22 (1971), 545-555.

24. P. Wilker, Adjoint product and hom functors in general topology, Pacific J. Math., 34 (1970), 269-283.

25. O. Wyler, convenient Categories for topology, General Topology and its Appl., 3 (1973), 225-242.

Received October 2, 1978 and in revised form June 29, 1979.

MeMORIAL UNIVERSITY OF NeWFOUNDLAND

St John's, Newfoundland, A1B 3X7, Canada 



\section{PACIFIC JOURNAL OF MATHEMATICS}

\section{EDITORS}

DONALD BABBITT (Managing Editor)

University of Galifornia

Los Angeles, California 90024

Hugo Rossi

University of Utah

Salt Lake City, UT 84112

C. C. MOORE AND ANDREW OGG

University of California

Berkeley, CA 94720
J. DugundjI

Department of Mathematics University of Southern California Los Angeles, California 90007

R. Finn and J. Milgram Stanford University Stanford, California 94305

ASSOCIATE EDITORS

E. F. BECKENBACH

B. H. NeumanN

F. WOLF

K. YoSHIDA

\section{SUPPORTING INSTITUTIONS}

UNIVERSITY OF BRITISH COLUMBIA

UNIVERSITY OF SOUTHERN CALIFONIA

CALIFORNIA INSTITUTE OF TECHNOLOGY

UNIVERSITY OF CALIFORNIA

MONTANA STATE UNIVERSITY

STANFORD UNIVERSITY

UNIVERSITY OF HAWAII

UNIVERSITY OF NEVADA, RENO

UNIVERSITY OF TOKYO

NEW MEXICO STATE UNIVERSITY

UNIVERSITY OF UTAH

OREGON STATE UNIVERSITY

WASHINGTON STATE UNIVERSITY

UNIVERSITY OF OREGON

UNIVERSITY OF WASHINGTON 


\section{Pacific Journal of Mathematics \\ Vol. 88, No. $1 \quad$ March, 1980}

Michael James Beeson, Extensionality and choice in constructive mathematics .................................... 1

José L. Blasco Olcina, Two questions on Wallman rings.............. 29

Peter I. Booth and J. Tillotson, Monoidal closed, Cartesian closed and convenient categories of topological spaces .................. 35

Peter B. Borwein, Rational functions with positive coefficients, polynomials and uniform approximations ......................... 55

Josip Globevnik, Fourier coefficients of the Rudin-Carleson extensions . . . . 69

Jacob Kofner, Quasimetrizable spaces ...................... 81

Mark Mandelker, Resolutions on the line ..................... 91

Lynn McLinden, An analogue of Moreau's proximation theorem, with application to the nonlinear complementarity problem ............ 101

Atsushi Murase, On the uniform distribution property of certain linear algebraic groups ................................ 163

Nicholas Th. Varopoulos, Zeros of $H^{p}$ functions in several complex

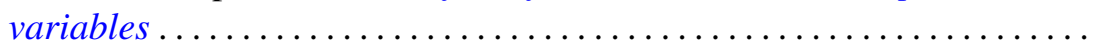

